\title{
La piézographie : étude comparative entre tests phonétiques arabe et français
}

\section{RÉSUMÉ}

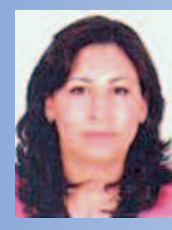

Rajae ZEROUAL

Professeur agrégée

de prothèse maxillo-faciale,

Université Hassan II,

Faculté de médecine dentaire

de Casablanca,

Rue Abou Alaa Zahar,

Quartier des hôpitaux,

BP 9157,

Casablanca, Maroc

\section{Abderrahman ANDOH}

Professeur de l'enseignement supérieur,

Biologie et matières fondamentales,

Université Hassan II,

Faculté de médecine dentaire

de Casablanca.

\section{Khadija KAOUN}

Professeur de l'enseignement supérieur de prothèse amovible,

Chef de département de prothèse amovible, Université Hassan II,

Faculté de médecine dentaire

de Casablanca.

Le modelage fonctionnel de l'espace prothétique par la méthode piézographique permet la confection de prothèses adjointes totales avec des extrados adaptés à la fonction des organes paraprothétiques.

Le but de cet article est d'étudier l'impact de la linguistique sur le modelage des empreintes fonctionnelles piézographiques chez l'édenté total. La méthode phonétique élaborée dans cette étude est basée sur l'exploitation des facteurs musculaires avec une variable linguistique. Deux empreintes piézographiques ont été réalisées, I'une utilisant des phonèmes français et l'autre des phonèmes arabes. Ensuite, des clefs en silicone ont été réalisées pour délimiter les couloirs prothétiques et servir par la suite de matériel de comparaison. Les contours des couloirs prothétiques des deux empreintes ont été marqués et superposés avec un crayon sur papier graphique.

- piézographie

- linguistique

- édentation totale
AOS 2012;257:63-71

DOI: $10.1051 / a 0 s / 2012108$

(C) EDP Sciences 


\section{Introduction}

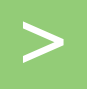

La piézographie en phonation est le résultat de l'activité synergique de deux pôles musculaires antagonistes : la langue et la sangle buccinato-labiale [1,2].

En confiant le modelage de l'empreinte aux organes paraprothétiques qui entrent en jeu au cours de la phonation, on élimine toute

\section{Matériel et patient}

Un patient sain, édenté total, âgé de 72 ans, présentant un stade avancé de résorption mandibulaire et ne présentant aucune pathologie de l'articulation temporo-mandibulaire, a été inclus dans cette étude (fig. 1).

\section{La base piézographique}

Comme la piézographie se fait en plusieurs temps, il nous a semblé nécessaire de réunir nos intervention manuelle de la part de l'opérateur et, par conséquent, tous les effets secondaires, artificiels et nocifs que cela implique. De plus, pour obtenir un résultat satisfaisant, il est indispensable que cette phonation soit minutieusement contrôlée par le praticien [3-5].

enregistrements à l'aide d'une base en résine acrylique qui a été réduite au strict minimum, afin de ne pas interférer avec le jeu des organes paraprothétiques.

Cette base a été utilisée, après avoir satisfait en bouche, les tests de Herbst [6], les tests phonétiques de Devin [7] et le patient a pu parler avec aisance sans que cette base ne quitte son siège et sa surface d'appui. Un duplicata conforme de cette base a été réalisé, pour servir de gabarit à

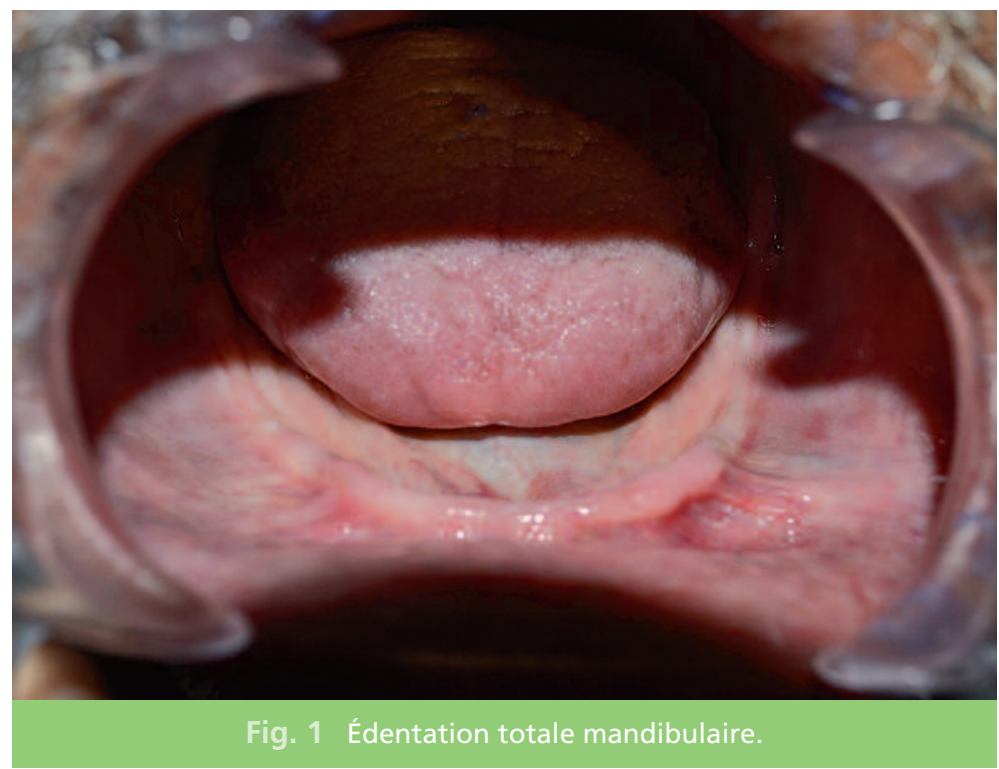


la prise de la deuxième empreinte piézographique [8].

\section{Le matériau}

Le matériau idéal doit répondre aux critères prérequis de stabilité et de fidélité. II doit, en outre, présenter un temps de plasticité suffisamment lent pour permettre les diverses manipulations [9-11]. En l'occurrence, dans notre cas, un thiocol dense (type Permlastic ${ }^{\circledR}$ regular de Kerr) a été utilisé.

\section{Les phonèmes utilisés}

Les phonèmes choisis pour le modelage doivent à la fois solliciter l'activité de deux pôles musculaires antagonistes : la langue et la sangle buccinato-labiale $[1,7,12]$.

\section{> Phonèmes français}

L'activation du modelage en phonation avec les phonèmes français, a été réalisée selon la séquence suivante, préconisée par Samoian [1, 2,9] (fig. 2).

- Pour la région buccinatrice, les phonèmes " SIS " répétés six fois et " SO " une fois.
- Pour la région antérieure de la mandibule :

- l'action centrifuge de la langue est obtenue par l'émission du «TE » et « DE »;

- l'action centripète provient des lèvres au moment de l'émission du « SE », « ME » et « $P E$ ».

\section{> Phonèmes arabes}

La langue arabe offre une situation de diglossie très caractérisée. Pour tout arabophone, la maîtrise de l'arabe classique résulte d'une acquisition qui se superpose au dialectal sans le supprimer [13-15].

L'alphabet arabe est formé de $\mathbf{2 8}$ graphèmes consonantiques (tableau I) [16].

L'arabe s'écrit de droite à gauche. Les lettres se lient entre elles comme dans l'écriture cursive du français ou de l'anglais. L'écriture d'imprimerie est identique à l'écriture manuscrite. On emploie les mêmes signes de ponctuation qu'en français mais on les écrit généralement à l'envers $[14,17]$.

Dans notre cas et dans un contexte d'analphabétisme, nous n'avons pas trouvé mieux que le verset coranique "Al-Nas " caractérisée par l'apparition répétitive de plusieurs sifflantes tout au long du verset (fig. 3) [18].

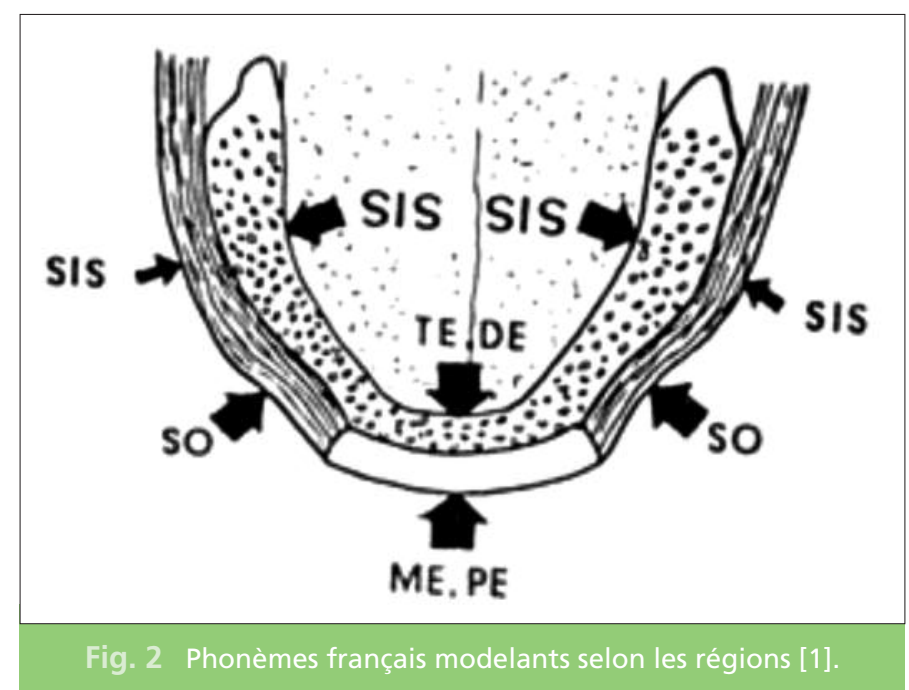




\begin{tabular}{|c|c|c|c|c|c|c|}
\hline \multicolumn{7}{|c|}{ الأبجدية اللعربية ; L'alphabet arabe } \\
\hline $\mathrm{N}^{\circ}$ & Lettre & Nom français & $\mathrm{T} 1$ & $\mathrm{~T} 2$ & Son & Nom arabe \\
\hline 0 & $\varepsilon$ & Hamza & , & 2 & arrêt sec & همزة \\
\hline 1 & 1 & Alif & a & A/â & a long & أنف \\
\hline 2 & $ب$ & $\mathrm{Ba}$ & $b$ & $\mathrm{~b}$ & $b$ & باء \\
\hline 3 & $ت$ & $\mathrm{Ta}$ & $\mathrm{t}$ & $\mathrm{t}$ & $\mathrm{t}$ & تناء \\
\hline 4 & $\stackrel{*}{*}$ & Sa & s & th & s lingué (à l'anglaise) & نثاء \\
\hline 5 & 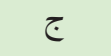 & Djim & j & j & $\mathrm{dj}$ & جيم \\
\hline 6 & $\tau$ & Hha & $\mathrm{h}$ & 7 & h expiré & حاء \\
\hline 7 & $\dot{\tau}$ & Kha & $\mathrm{h}$ & 5 & h expiré et raclé & خاء \\
\hline 8 & د & Del & d & d & $d$ & دان \\
\hline 9 & j & Zel & $d$ & $\mathrm{dh}$ & z lingué & ذان \\
\hline 10 & $\jmath$ & $\mathrm{Ra}$ & $r$ & $\mathrm{R}$ & r roulé & راء \\
\hline 11 & j & Zay & z & z & z & زاي \\
\hline 12 & س س & $\operatorname{Sin}$ & s & $\mathrm{s}$ & $s$ & سبن \\
\hline 13 & ش ش & Shin & sh & $\mathrm{ch}$ & $\mathrm{ch}$ & شين \\
\hline 14 & ص & Sad & s & $\mathrm{S}$ & s emphatique (plus ou moins appuyé) & صناد \\
\hline 15 & 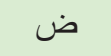 & Dad & d & $\mathrm{D}$ & d emphatique (plus ou moins appuyé) & 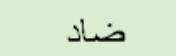 \\
\hline 16 & b & $\mathrm{Ta}$ & $\mathrm{t}$ & $6 / \mathrm{T}$ & t emphatique ( appuyé) & طله \\
\hline 17 & 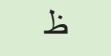 & $\mathrm{Za}$ & z & Z & z emphatique ( appuyé) & ظلاء \\
\hline 19 & $\varepsilon$ & Ayn & , & $3 /{ }^{\prime}$ & coup de glotte & 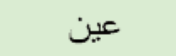 \\
\hline 18 & $\dot{\varepsilon}$ & Rayn & r & $\mathrm{r} / \mathrm{gh}$ & r à la Parisienne & 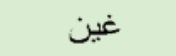 \\
\hline 20 & ف & $\mathrm{Fa}$ & $f$ & $f$ & $f$ & فاء \\
\hline 21 & ق & Qaf & $q$ & $9 / q$ & k emphatique (plus ou moins appuyé) & 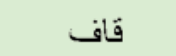 \\
\hline 22 & 5) & Kaf & k & $\mathrm{k}$ & $\mathrm{k}$ & كاف \\
\hline 23 & J & Lam & 1 & I & 1 & لام \\
\hline 24 & 5 & Mim & $\mathrm{m}$ & $\mathrm{m}$ & $\mathrm{m}$ & 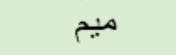 \\
\hline 25 & $\dot{ن}$ & Noun & $\mathrm{n}$ & $\mathrm{n}$ & $\mathrm{n}$ & ن \\
\hline 26 & ○ & $\mathrm{Ha}$ & $\mathrm{h}$ & $\mathrm{h}$ & h expiré (à l'anglaise) & هlء \\
\hline 27 & 9 & Waw & w & $w$ & û long / w & 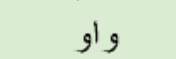 \\
\hline 28 & ي & Ya & y & y/î & î long / y & باء \\
\hline
\end{tabular}

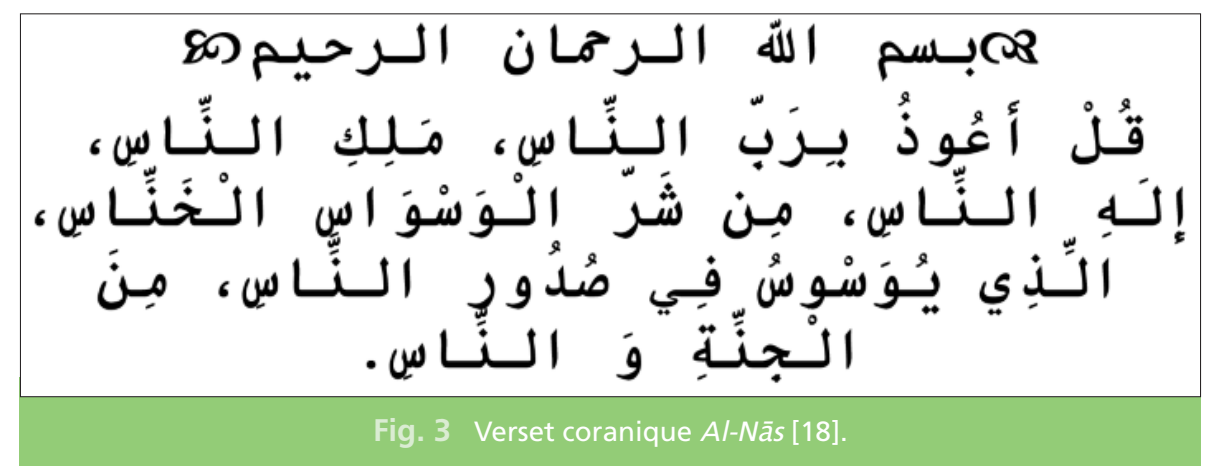




\section{L'empreinte piézographique phonétique}

\section{> Préempreinte issue des phonèmes français}

La base en résine préalablement adaptée et préformée selon la configuration de l'arcade, est enrobée de matériau. Elle est placée en bouche et le patient est invité à s'animer pendant une dizaine de minutes, selon la séquence des phonèmes français citée ci-dessus.

L'empreinte obtenue après ce premier enregistrement est appelée : préempreinte avec phonèmes français.

\section{> Préempreinte issue des phonèmes arabes}

Le duplicata de la base en résine est enrobé du même matériau et, de la même façon que précédemment, le même patient est invité à réciter le verset coranique "Al-Nas » pendant une dizaine de minutes : I'empreinte obtenue après ce deuxième enregistrement est appelée préempreinte avec phonèmes arabes.

\section{$>$ Remarginage des préempreintes}

Les préempreintes ainsi obtenues sont remarginées avec le même matériau et introduites en bouche et le patient est prié de reprendre la même séquence d'exercices phonatoires de la même manière qu'il en a été informé au préalable : les phonèmes français seront utilisés en premier pour remarginer la première préempreinte et dans un second temps, les phonèmes arabes seront utilisés pour remodeler le remarginage de la deuxième préempreinte (fig. 4 et 5).

Ces opérations seront répétées autant de fois qu'il est jugée nécessaire. Enfin, on procèdera à un rajout de matériau jusqu'à atteindre le volume prothétique escompté.

\section{Rebasage}

Quand le volume prothétique est jugé satisfaisant, les empreintes sont récupérées pour être rebasées à l'aide d'un thiocol de faible viscosité (type Permlastic ${ }^{\circledR}$ light de Kerr).

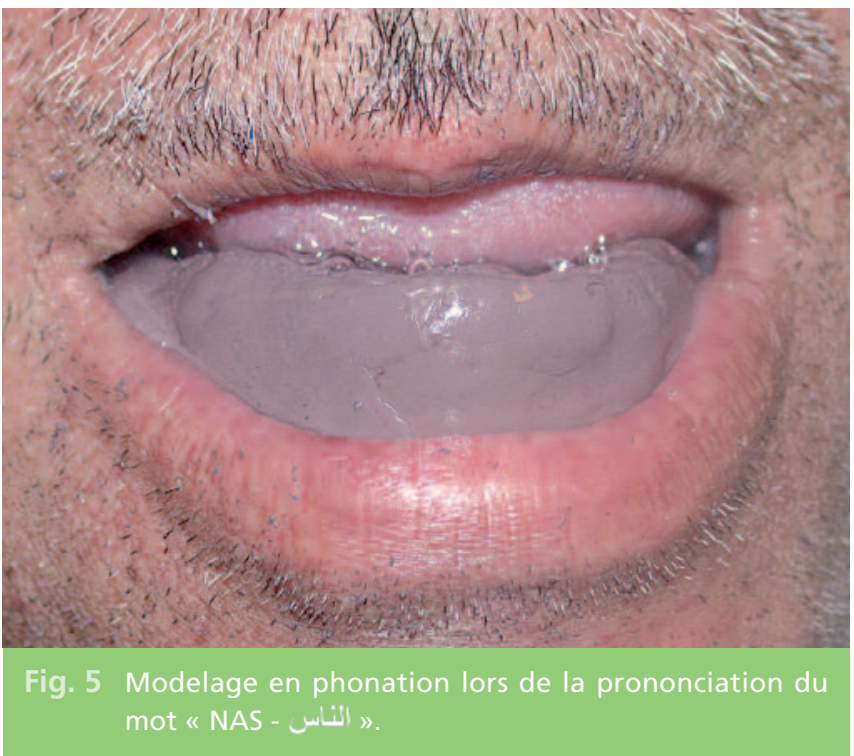




\section{Réalisation des clefs de la piézographie}

Les empreintes piézographiques ainsi rebasées sont coffrées et coulées avec un plâtre dur. Avant de démouler les empreintes, deux clefs

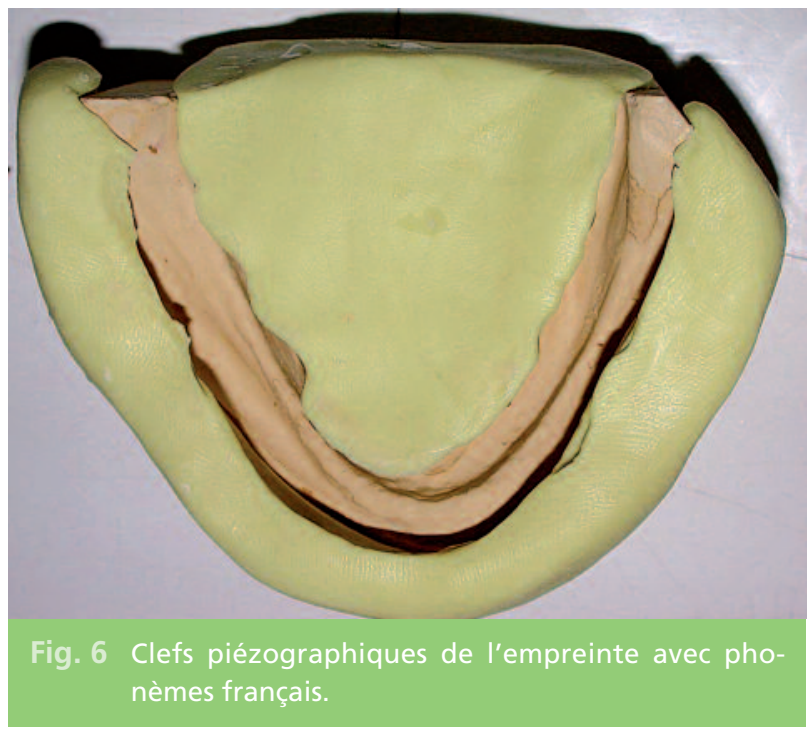

\section{Résultats}

Les clefs en silicone sont récupérées pour servir de matériel de comparaison, elles ont servi à délimiter les couloirs prothétiques des deux empreintes piézographiques, obtenues successivement, l'une en utilisant les phonèmes français, et l'autre, les phonèmes arabes.

Les contours des couloirs prothétiques matérialisés par les clefs en silicone sont reproduits à l'aide d'un crayon sur un papier graphique (fig. 8).

La superposition des tracés des couloirs prothétiques déterminés par les méthodes phonétiques arabe et française a montré des différences au niveau des régions prémolaires droite vestibu- en silicone vestibulaire et linguale sont confectionnées, pour chacune des deux empreintes. Les faces supérieures de ces clefs doivent être alignées sur le plan occlusal représenté par la face supérieure de la piézographie (fig. 6 et 7).

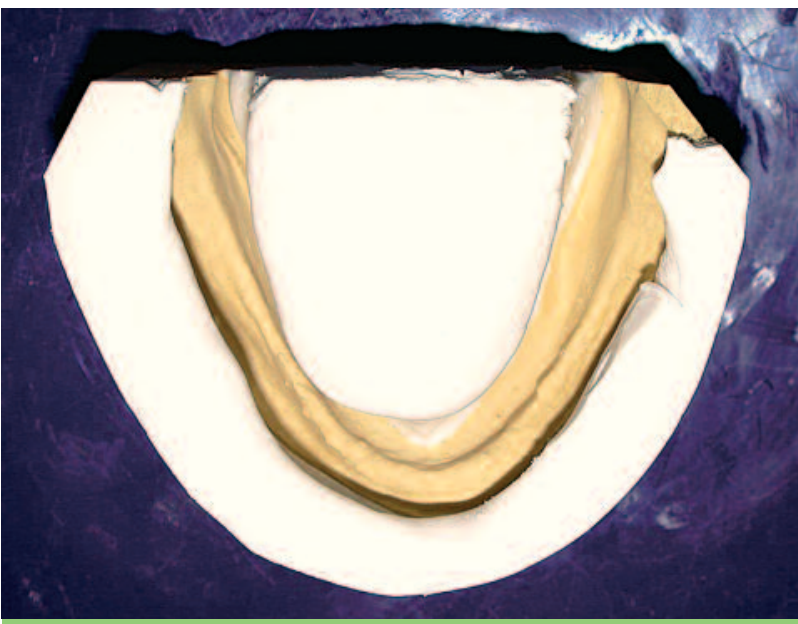

Fig. 7 Clefs piézographiques de l'empreinte avec phonèmes arabes.

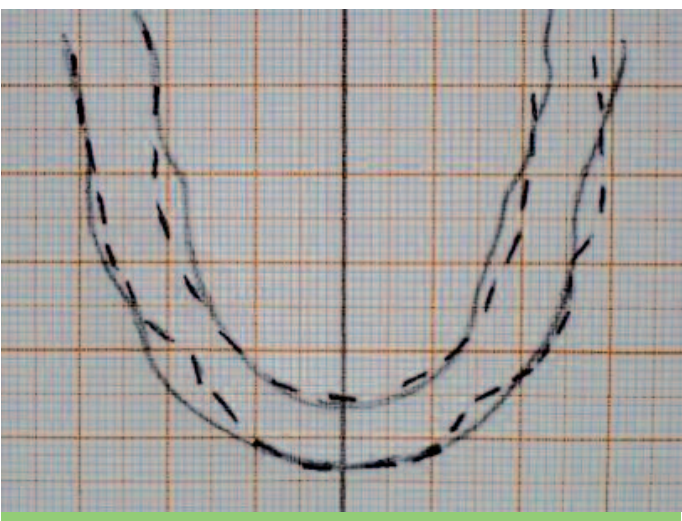

Fig. 8 Superposition des tracés des couloirs prothétiques déterminés par les méthodes phonétiques arabe et française. La ligne continue représente la méthode phonétique française, la ligne discontinue représente la méthode phonétique arabe. 
laire $(4 \mathrm{~mm})$ et de la région molaire droite linguale ( $3 \mathrm{~mm}$ ). Des différences ont été également notées au niveau de la région prémolaire gauche vestibulaire $(2 \mathrm{~mm})$ et de la région molaire gauche vestibulaire et linguale ( $2 \mathrm{~mm}$ ).

Au niveau de la région antérieure, il n'y a pas de différence entre les deux couloirs prothétiques.

\section{Discussion}

Cette étude n'a pas été conçue pour déterminer laquelle des deux langues, arabe ou française, est meilleure pour la stabilité d'une prothèse adjointe complète. Le seul objectif étant d'évaluer s'il y a une différence significative entre les couloirs prothétiques déterminés par les deux empreintes phonatoires.

L'analyse des contours des couloirs prothétiques des empreintes piézographiques arabe et française indique des différences au niveau des régions prémolo-molaires droite et gauche. En

\section{Conclusion}

Cette étude a utilisé deux empreintes piézographiques phonétiques. Le résultat obtenu doit être considéré avec prudence sans, toutefois, le généraliser à tous les patients. D'autres études doivent être réalisées dans ce sens pour randomiser le résultat obtenu.

Une étude validée par des phonèmes arabes puis français sollicitant l'activité des deux pôles
Dans les limites de cette étude, le couloir prothétique délimité par l'empreinte phonétique arabe semble être plus étroit au niveau postérieur par rapport à celui issu de l'empreinte phonétique française, ce qui peut limiter l'espace dévolu au positionnement des prémolaires et molaires à ce niveau.

effet, celui issu de l'empreinte phonétique arabe est plus étroit. En conséquence, les dents seront placées dans un espace plus limité dans le sens vestibulo-lingual.

Deux facteurs peuvent être responsables de ce résultat :

- soit le composé est trop visqueux et a été suffisamment moulé par le muscle buccinateur ; - soit le muscle buccinateur a une activité modelante augmentée avec les phonèmes arabes.

musculaires antagonistes, la langue et la sangle buccinato-labiale, impliqués dans l'enregistrement de l'empreinte piézographique, doit être réalisée sur plusieurs patients afin de déterminer l'influence de la linguistique sur l'activité modelante des muscles circum-prothétiques. 


\section{Bibliographie}

1. Samoian $\mathrm{R}$.

Apport des techniques

piézographiques

en prothèse adjointe totale.

Act Odonto Stomatol

1992;117:157-77.

2. Tondowski E.

L'influence des remparts

prothétiques

sur la phonétique

en prothèse adjointe totale.

Stratégie Prothétique

2002;2(5):371-82.

3. Tondowski E.

L'empreinte phonétique

en prothèse totale.

Stratégie Prothétique

2004;4(2):107.

4. Beresin VE, Schiesser FJ.

The neutral zone

in complete dentures.

J Prosthet Dent

2006;95:93-100.

5. Alfano SG, Leupold RJ.

Using the neutral zone

to obtain

maxillomandibular

relationship records

for complete denture

patients.

J Prosthet Dent

2001;85:621-3.

6. Gilbert $\mathrm{Y}$, Soulet $\mathrm{H}$,

Blandin $\mathrm{M}$.

Phénomènes rétentifs

en prothèse adjointe

complète.

Encycl Méd Chir

Stomatologie

1987:23325-B-056.
7. Nabid A, Klein P.

Méthodologie du moulage

de l'espace prothétique

mandibulaire

gérodontologique :

technique phonétique.

Odontologia 1984;6:31-44.

8. Ikebe K, Okuno I, Nokubi T. Effect of adding

impression material

to mandibular denture

space in piezography.

J Oral Rehabil

2006;33(6):409-415.

9. Jardel V, Richard A,

Hirigoyen M.

Les empreintes

piézographiques : évolution

dans le choix des matériaux.

Cah Prothèse 1992;79:27-35.

10. Makzoumé JE.

Morphologic comparison of

two neutral zone

impression techniques:

a pilot study.

J Prosthet Dent

2004;92(6):563-568.

11. Bouetel $B$, Le Bars $P$,

Bremont JF, Giumelli B.

Complémentarité

des techniques implantaires

et piézographiques

en prothèse amovible

complète.

Cah Prothèse

2007;139:61-9.

12. Giovanni A, Ouaknine M,

Garrel R.

Physiologie

de la phonation.
Encycl Méd Chir

2003;20-632-A-10.

13. Essono JM.

Précis de linguistique

générale.

Paris : Éditions l'Harmattan, 1998.

14. El Shafei $M$, Al-Muhtaseb $H$, Al-Ghamdi $\mathrm{M}$.

Techniques for high quality arabic speech synthesis.

Information Sciences

2002;140(3-4):255-267.

15. Kirchoff K, Vergyri D.

Cross-dialectal data sharing for acoustic modelling

in Arabic speech

recognition.

Speech Communication

2005;46:37-51.

16. Boukadida N.

Connaissances

phonologiques

et morphologiques

dérivationnelles

et apprentissage

de la lecture en arabe

(étude longitudinale).

Thèse Doct Psycho.

Rennes: Univ européenne de Bretagne, 2008.

17. Delavaux V, Demolin D,

Harmegnies B, Soquet A.

The aerodynamics of

nasalization in French.

J Phonetics

2008;36:578-606.

18. Sūrat Al-Nās.

(Arabic: سورة الناس ).

en.wikipedia.org/wiki/Surat_al-Nas 


\section{SUMMARY}

\section{Piezography : comparative study between tests using French phonemes and Arabic phonemes}

Rajae ZEROUAL, Abderrahman ANDOH, Khadija KAOUN

\section{Keywords} - piezography - linguistic - total edentation
The functional modelling of prosthetic space by the piezographic method allows making a total prosthesis with adapted polished surfaces to the function of the paraprosthetic organs.

The purpose of this article is to study the impact of the linguistic on the modelling of the functional piezography impression in complete denture. The phonetic method elaborated in this study is based on the exploitation of the muscular factors with a linguistic variable.

Two piezography impressions were realized, the one using French phonemes and the other using Arabic phonemes. Then, two silicon keys were realized to bound prosthetic corridors and to serve afterward as a material of comparison. The outlines of both impression corridors were marked and superposed with a pencil on a graphic paper.

\section{Questions-Réponses}

\section{> 1. - QCM à une seule réponse :}

\section{La base piézographique :}

a-Ne doit pas satisfaire aux tests de Herbst.

$\square b$ - Permet de réunir les différents enregistrements piézographiques.

$\square$ c - Ne peut pas être en résine acrylique.

$\mathrm{d}$ - Peut interférer avec le jeu des organes paraprothétiques.

$$
\text { q - }\llcorner: \text { : əsuodọy }
$$

\section{> 2. - Questions-réponses:}

a - La piézographie en phonation ne peut pas être le résultat de l'activité de deux pôles musculaires antagonistes:

\section{$\square$ VRAIX $\square$ FAUX}

b - La piézographie en phonation élimine toute intervention manuelle de la part de I'opérateur :

\section{$\square$ VRAIX $\square$ FAUX}

$I \forall y \Lambda: q-乙-X \cap \forall\rfloor: e-Z:$ səsuodə̨y 